\title{
DESENVOLVIMENTO DE SENSORES DE BAIXO CUSTO PARA DETECÇÃO DE AROMAS ALIMENTÍCIOS COMERCIAIS
}

\author{
L. TIGGEMANN $^{(1)}$, C. STEFFENS ${ }^{(2)}$, E. VALDUGA $^{(2)}$, J. STEFFENS $^{(2)}$, A. MANZOLI $^{(3)}$,

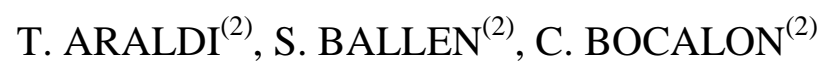 \\ (1) Universidade Federal de Santa Catarina, Departamento de Engenharia Química e de Alimentos; \\ ${ }^{(2)}$ Universidade Regional Integrada do Alto Uruguai e das Missões - Campus Erechim, \\ Departamento de Engenharia de Alimentos; \\ ${ }^{(3)}$ Embrapa Instrumentação, Laboratório Nacional de Nanotecnologia para o Agronegócio.
}

E-mail: liditiggemann@yahoo.com.br

RESUMO- O presente trabalho teve por objetivo desenvolver sensores de gases por meio da técnica de formação de trilhas, usando como substrato o papel vegetal para detecção de aromas comerciais alimentícios. O sensor de gás mostrou boa sensitividade aos aromas de morango A, uva, abacaxi e maça e baixa sensitividade aos aromas de cereja e morango B, variando de 16-125\%. A influência de diferentes dopantes, ácido clorídrico (HCI), ácido canforsulfônico (CSA) e dodecil benzeno sulfônico (DBSA), na sensitividade, no tempo de resposta do sensor e na morfologia superficial do filme de Pani também foi investigada. A melhor sensitividade (112\%) e os menores tempos de resposta ( $3 \mathrm{~min}$ ) foram encontrados para os sensores dopados com $\mathrm{HCl}$. O filme de Pani dopado com $\mathrm{HCl}$ apresentou melhor regularidade superficial, com partículas menores e melhor distribuídas

Palavras-chave: sensores de gás, aromas comerciais, polianilina, técnica de formação de trilhas, sensitividade.

\section{INTRODUÇÃO}

A identificação e a quantificação de aromas tem atraído muito a atenção do corpo científico e dos setores econômicos, pois os mesmos são normalmente utilizados na indústria química e indústria farmacêutica (por exemplo, antibióticos, antissépticos, anti-inflamatórios e agentes antiparasitários), no setor de alimentos (por exemplo, aromatizantes) e na indústria de cosmético (por exemplo, perfumes) (Biniecka; Caroli, 2011).

A abordagem clássica para a avaliação de aromas alimentícios é baseada na análise sensorial, ou seja, a análise que emprega o uso dos sentidos (sabor, aroma, visão e tato) realizado por um grupo de pessoas devidamente treinado. A análise sensorial pode ser uma ótima ferramenta na realização de testes de preferência dos consumidores, mas contêm muitas limitações: é um método caro, demorado, limitado a compostos que não sejam tóxicos e sofre de incoerência e imprevisibilidade devido a vários fatores humanos (variabilidade individual, diminuição da sensibilidade devido à prolongada exposição, fadiga e estados mentais variáveis) (Banerjee et al., 2012). 
Devido às deficiências dos métodos de análise sensorial, um complemento na avaliação organoléptica dos alimentos é a análise instrumental. Análises instrumentais utilizando técnicas adequadas permitem uma análise qualitativa detalhada e análises quantitativas dos componentes voláteis que compõem os aromatizantes dos produtos alimentares. A técnica instrumental mais comum para avaliação de aromas é a cromatografia gasosa acoplada à espectrometria de massa (GC-MS) (Wyllie, 2008).

Porém a complexidade da maioria dos aromas de alimentos, alguns contendo dezenas de compostos diferentes dos quais apenas uma parte é percebida pelos sentidos humanos, dificultam sua caracterização por técnicas de cromatografia gasosa. Além disso, o custo e o consumo de energia desta técnica muitas vezes são incompatíveis com os requisitos do produto.

Assim, uma alternativa é o emprego de sensores químicos de baixo custo que apresentem uma resposta rápida e confiável para a detecção de compostos voláteis presentes nos alimentos (Torri et al., 2010). Os sensores disponíveis no mercado são fabricados em sua maioria com semicondutores inorgânicos, como o óxido de estanho. Apesar desses sensores serem baratos, eles são pouco seletivos. Com isso, novas alternativas vêm sendo investigadas na área de sensores, em particular de gases, e nesse contexto moléculas orgânicas têm sido exploradas, dentre as quais se destacam os polímeros condutores (Guiseppi-Elie et al., 1998). $\mathrm{O}$ interesse em polímeros condutores se deve ao fato de que na presença de um gás sua condutividade elétrica pode ser acentuadamente alterada, e esta mudança pode então ser precisamente detectada (Steffens, 2009).

A polianilina (Pani) é um dos polímeros que vêm sendo amplamente estudado, pois apresenta facilidade de polimerização e dopagem, boa estabilidade química ambiental e baixo custo. Desta maneira, várias aplicações tecnológicas têm se utilizado desse material para produzir sensores, entre outros dispositivos (Li et al., 2013).

O desenvolvimento de tecnologia barata e "descartável" de sensor é muito importante, uma vez que estes podem ser utilizados para diversas aplicações, como por exemplo, no desenvolvimento de biossensores, narizes eletrônicos e línguas eletrônicas (Venancio et al., 2008). A técnica de formação de trilhas sobre papel vegetal merece destaque e requer baixo investimento. O papel vegetal pode ser de fácil manipulação, é biodegradável menos nocivo para o ambiente que outros sensores disponíveis (que empregam vidro, PET como substrato).

Dentro deste contexto, o trabalho teve por objetivo desenvolver sensores de gases por meio da técnica de formação de trilhas, usando como substrato o papel vegetal para detecção de aromas comerciais alimentícios.

\section{MATERIAL E MÉTODOS}

\subsection{Elaboração dos sensores de gases}

Os eletrodos interdigitados a base de grafite foram produzidos pelo uso da técnica de formação de trilhas como o descrito por Steffens (2010). Após, filmes finos de Pani foram depositados nos eletrodos interdigitados pela técnica de polimerização in-situ conforme Steffens (2009). 


\subsection{Aparato experimental}

Para realização dos experimentos de detecção de aromas comerciais empregando sensores de gases com filme de Pani um aparato experimental foi construído (Figura 1). O aparato experimental utilizado é constituído por: câmara de vidro com capacidade de $2,15 \mathrm{~L}$ (1), tampa de vedação (2), reservatório para o aroma (3), termo-higrômetro (IMIMIPA MT241) (4), multímetro (Fortools-16517) (5) e temporizador digital (Cronobio - SW2018) (6). Os sensores de gás foram fixados no interior da câmara (um por vez) e, inicialmente, uma linha de base foi obtida. Após aquisição os dados foram tabelados e tratados com auxílio de software estatístico (Statistica 7.0).

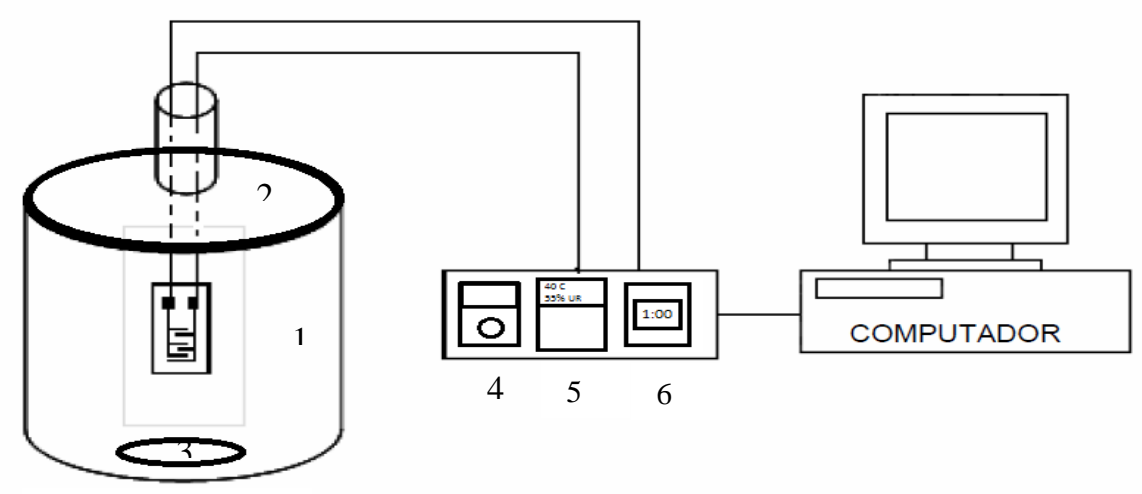

Figura 1 - Representação esquemática do aparato experimental

\subsection{Monitoramento da resistência elétrica dos sensores de gases}

Antes da realização dos experimentos de detecção dos aromas a resistência elétrica dos sensores foram obtidas a cada $30 \mathrm{~s}$ durante $15 \mathrm{~min}$ para aquisição de linha de base. Esta medida foi obtida por meio de um multímetro (FLUKE, modelo 77III). Também em cada análise monitorou-se a temperatura e a umidade com o auxílio de um sensor termo-higrômetro (IMIMIPA MT-241). Este monitoramento foi efetuado para verificar o comportamento do filme de Pani sobre os sensores nas condições ambientes.

\subsubsection{Detecção dos aromas comerciais com os sensores de gases}

Após aquisição de linha base, iniciaram-se os experimentos para avaliação da detecção dos sensores com filmes de Pani a diferentes aromas comerciais. Os experimentos foram realizados em duas etapas: na primeira etapa os sensores foram expostos ao aroma de interesse no interior da câmara durante $15 \mathrm{~min}$, sendo que aquisição da resistência foi efetuada a cada minuto e, na segunda etapa os sensores foram novamente expostos às condições ambientes (ar de laboratório) durante $15 \mathrm{~min}$. A temperatura e a umidade foram medidas a cada minuto em cada uma das etapas citadas acima. Esse procedimento foi repetido por mais dois ciclos.

Os sensores foram expostos a $5 \mathrm{~mL}$ de diferentes aromas (morango $\mathrm{A}$, morango $\mathrm{B}$, uva, maçã, abacaxi e cereja). A sensitividade e a reversibilidade dos sensores ao aroma analisado foi calculada pelas Equações 1 e 2. 


\subsubsection{Avaliação da sensitividade e reversibilidade da resposta dos sensores na presença de um determinado aroma}

O parâmetro reversibilidade $(\eta)$ descreve a capacidade dos sensores para voltar ao seu estado inicial depois de ter sido exposto a um gás em particular.

A sensitividade $(S)$ e a reversibilidade $(\eta)$ de cada um dos sensores foram calculadas como descrito nas equações (1) e (2), respectivamente.

$$
S=\frac{\left(r-r_{O}\right)}{r_{O}} 100
$$

onde: $\mathrm{r}_{\mathrm{o}}$ é a resistência inicial do sensor e $\mathrm{r}$ é valor da resistência após exposição ao aroma pelo tempo exposto.

$$
\eta=\frac{\left(r-r_{f}\right)}{\left(r-r_{O}\right)} 100
$$

onde: $r_{o}$ é a resistência inicial do sensor; $r$ é valor da resistência após exposição ao aroma pelo tempo exposto e $\mathrm{r}_{\mathrm{f}}$ é a resistência final do sensor.

\subsubsection{Respostas dos sensores a diferentes dopantes}

Os sensores de gases com filme de Pani dopados com $\mathrm{HCl}$ foram desdopados por imersão em solução 0,1 M de hidróxido de amônio $(\mathrm{NH} 4 \mathrm{OH})$ por $30 \mathrm{~s}$ e, redopado por $90 \mathrm{~s}$ em uma solução $0,1 \mathrm{M}$ do dopante investigado. Os ácidos de dopagem estudados foram o ácido clorídrico - $\mathrm{HCl}$, ácido canforsulfônico - CSA e ácido dodecilbenzeno sulfônico DBSA. Estes ácidos foram escolhidos em função de trabalhos relatados na literatura (Steffens et al., 2010; Pattananuwat e Aht-Ong, 2010)

Após aquisição de linha base os sensores contendo diferentes dopantes foram expostos durante $60 \mathrm{~min}$ ao aroma de interesse. A temperatura e a umidade foram monitoradas (a cada minuto) utilizando sensores comerciais de umidade e temperatura (Sensirum e IMIMIPA MT241) durante todo experimento.

Os sensores de gases com filme de Pani com diferentes dopantes foram avaliados em relação à sensitividade (Equação 1) e e o tempo de resposta. Segundo a Sensirion (2012) o tempo de resposta é um parâmetro muito importante para avaliar o desempenho dos sensores, o qual é definido como o tempo que um sensor leva para responder a presença de um COV. (considerando que o sensor estava em sua linha de base), para alcançar $63 \%$ do seu valor máximo estável.

\subsection{Microscopia eletrônica de varredura (MEV)}

A microscopia eletrônica de varredura (MEV) foi aplicada para a avaliação da morfologia superficial da Pani com diferentes ácidos dopantes (CSA, DBSA e HCl).

O princípio da Microscopia Eletrônica de Varredura é amplamente utilizado devido ao seu alto poder de resolução e grande profundidade de foco (300 vezes maior que o microscópio ótico). As imagens MEV são construídas ponto a ponto. 
As amostras dos sensores dopados com diferentes ácidos ( $\mathrm{HCl}$, DBSA e CSA) foram recobertos com uma camada ouro $(2 \mathrm{~nm})$ e caracterizados por microscopia eletrônica para determinação de tamanho e formato de partículas (Jeol JSM-6510)

\section{RESULTADOS E DISCUSSÃO}

A sensitividade (Figura 2-a) e a reversibilidade (Figura 2-b) dos sensores de gás foram avaliadas em triplicata. $\mathrm{O}$ sensor de gás mostrou boa sensitividade aos aromas de morango A, uva, abacaxi e maça e baixa sensitividade aos aromas de cereja e morango B, variando de 16$125 \%$. A umidade relativa permaneceu praticamente constante durante os experimentos (oscilação $\pm 2 \%$ ), demonstrando a eficiência do sensor na detecção dos diferentes aromas.

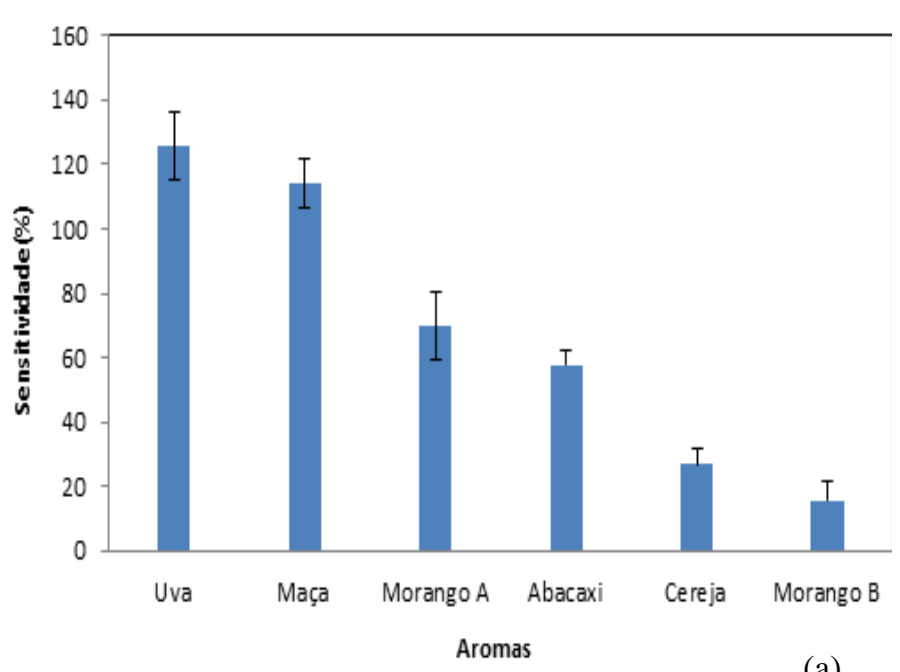

(a)

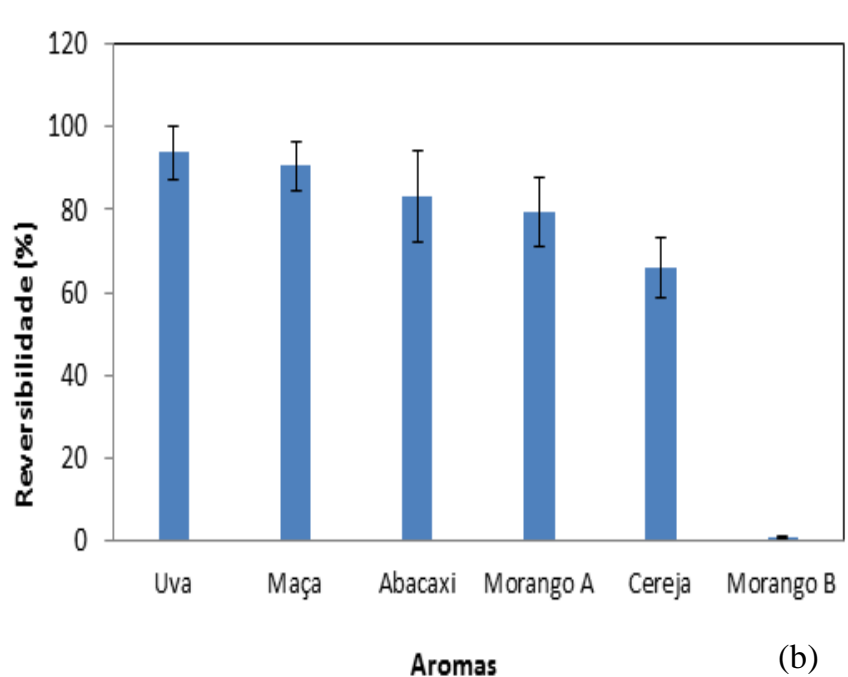

(b)

Figura 2 - Sensitividade e reversibilidade dos sensores de gases aos aromas testados.

Hawari et al., (2012) desenvolveram sensores baseados em microbalanças de quartzo para a detecção de voláteis durante a maturação da manga. Os autores encontraram sensitividade média de $83 \%$, inferior aos valores encontrados neste estudo para a análise dos aromas de uva, maçã e abacaxi empregando sensores com filmes de Pani.

Os aromas de cereja e morango $\mathrm{B}$, para os quais se obteve baixa sensitividade, estão diluídos em uma base oleosa, diferente dos demais aromas testados. A baixa sensitividade do sensor de gás na detecção destes aromas pode estar relacionada à base em que estes aromas estão diluídos. Uma modificação da composição dos alimentos pode favorecer ou dificultar a liberação de alguns compostos aromáticos. É sabido que a presença de gordura pode influenciar a liberação na fase vapor dos compostos que constituem o aroma (Voilley et al., 2006).

Lipídios são ingredientes que tem um grande impacto sensorial na percepção de aromas, eles diminuem a evaporação de vários compostos voláteis e influenciam o perfil aromático. $\mathrm{O}$ meio lipídico induz a uma redução na percepção dos constituintes do aroma devido também a consistência do seu meio (Relkin; Fabre, e Guichard, 2004). Justificando a menor sensitividade e reversibilidade detectada por meio dos sensores.

Neste estudo foi obtida uma boa reversibilidade para os sensores de gases quando expostos aos aromas de uva, maça, abacaxi e Morango A (80-93\%). Este resultado indica que 
para estes aromas os sensores desenvolvidos neste trabalho apresentam baixa perda de sinal, durante os sucessivos ciclos.

\subsubsection{Respostas dos sensores a diferentes dopantes}

A Figura 3 mostra a sensitividade dos sensores de gases com diferentes dopantes $(\mathrm{HCl}$, CSA e DBSA) na detecção dos aromas de morango, uva e maça.

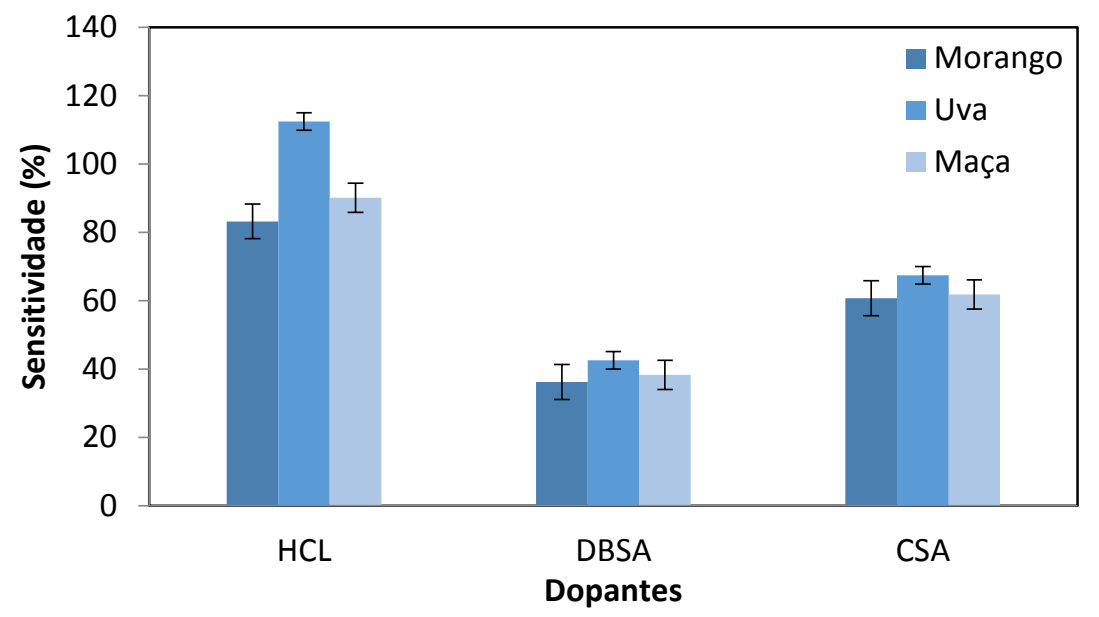

Figura 3. Sensitividade dos sensores de gases com diferentes dopantes (HCl, DBSA, CSA) aos aromas de morango, uva e maça.

Pode-se observar na Figura 3 que os sensores com diferentes dopantes diferiram amplamente em seus níveis de sensitividade em todos os aromas analisados. Os maiores valores de sensitividade foram encontrados para o dopante $\mathrm{HCl}$ (83-112\%), por outro lado os menores valores foram para o dopante DBSA (36-42\%). Dessa forma, a resposta desses sensores deve ser associada com tipo de dopante utilizado. Cada dopante proporciona um tipo de seletividade ao gás, o que na literatura tem sido chamado de reconhecimento molecular (Stefens, 2012).

O tipo de dopante, tamanho da molécula e processo de dopagem pode influenciar de forma significativa na sensitividade, tempo de recuperação e tempo de resposta de um sensor. Estudos realizados por Sengupta, Kar e Adhikari (2009), mostram a influência da utilização do ácido p-toluenossulfônico (PTSA), e do $\mathrm{HCl}$ no processo de dopagem de sensores de Pani na detecção de amônia. Os resultados demonstraram que o tempo de resposta e a sensitividade foram melhores para a Pani dopada com $\mathrm{HCl}$, pois o maior volume do grupo PTSA dificulta a absorção da amônia na estrutura.

A microscopia eletrônica de varredura (MEV) foi aplicada para a avaliação da morfologia superficial da Pani com diferentes ácidos dopantes (CSA, DBSA e $\mathrm{HCl}$ ) (Figura $4)$. 


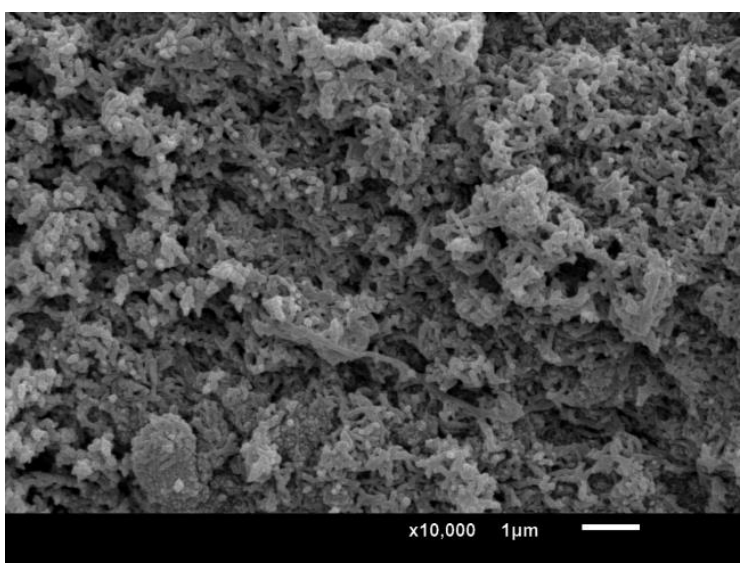

(a)

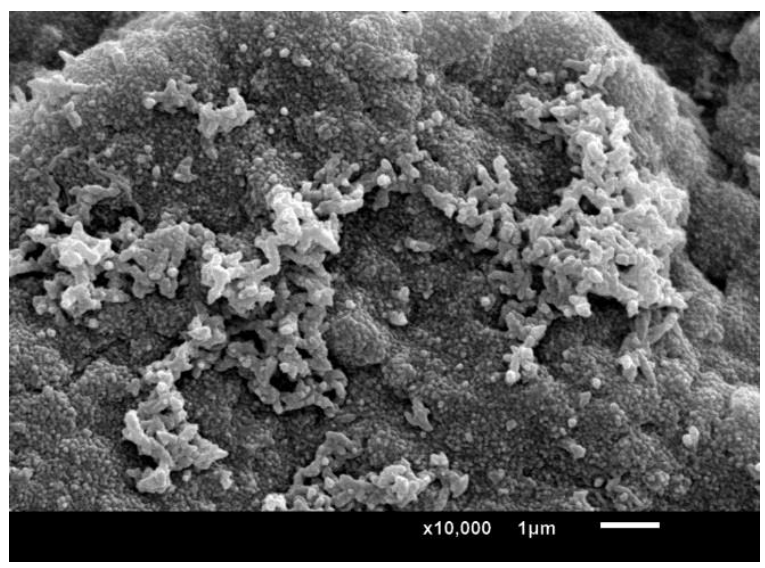

(b)

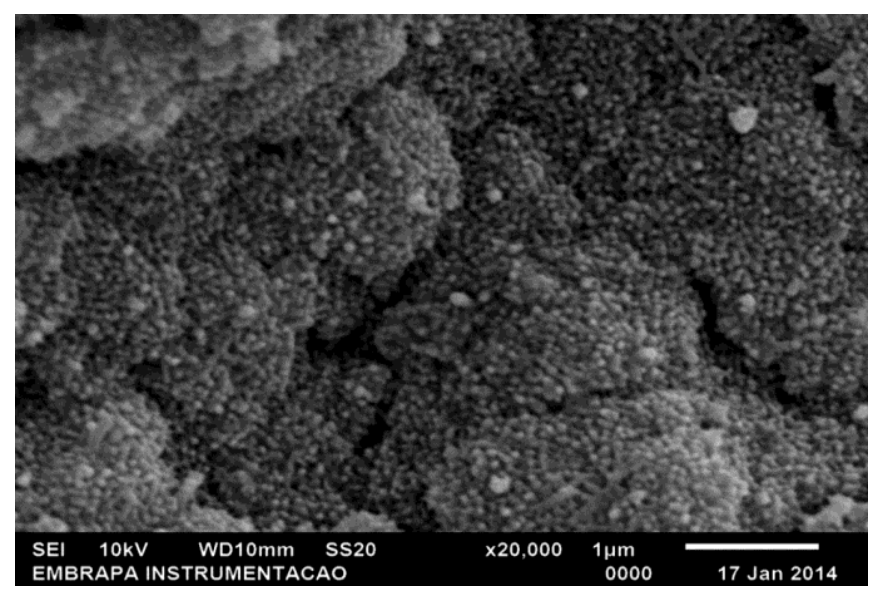

(c)

Figura 5. Micrografias da superfície do sensor com os diferentes dopantes: (a) CSA (b) DBSA e (c) HCL.

Pode-se observar que o tipo de dopante empregado proporcionou modificações no tamanho das partículas de Pani. O uso de $\mathrm{HCl}$, CSA e DBSA na dopagem da Pani, resultou em partículas aglomeradas, sendo que a maior aglomeração foi encontrada na Pani dopada com DBSA, o que pode ser atribuído à longa cadeia alifática do DBSA. Segundo Mattoso (1996) a morfologia da Pani está fortemente relacionada com a dopagem e o processamento.

Os menores tempos de resposta foram encontrados para os sensores dopados com $\mathrm{HCl}$ (3-11min). Segundo Weber et al., (2000) partículas consideravelmente menores, ou seja, com uma superfície de contato para o gás bem maior, tem um maior número de sítios ativos disponíveis, e consequentemente o tempo necessário para a interação entre o analito e a superfície é reduzido. Analisando a MEV para o filme Pani dopada com $\mathrm{HCl}$ é possível verificar que o filme de Pani dopada com $\mathrm{HCl}$ apresentou melhor regularidade superficial, com partículas menores e melhor distribuídos o que pode ter resultado em melhores valores de sensitividade para este dopante e menores tempos de resposta para detecção de todos os aromas analisados. 


\section{CONCLUSÕES}

O sensor de gás com filme de Pani mostrou boa sensitividade aos aromas de morango A, uva, abacaxi e maça e baixa sensitividade aos aromas de cereja e morango B, variando de 16-125\%. Os melhores resultados de sensitividade (112\%) e os menores tempos de resposta (3 min) em temperatura ambiente foram encontrados para os sensores dopados com $\mathrm{HCl}$. A morfologia do filme de Pani dopado com $\mathrm{HCl}$ apresentou melhor regularidade superficial, com partículas menores e melhor distribuídas.

\section{REFERÊNCIAS BIBLIOGRÁFICAS}

AKSU, Z.; EREN, A.T. Production of carotenoids by isolated yeast of Rhodotorula glutinis. Biochemical Engineering Journal. v. 35, p. 107 - 113, 2007.

BINIECKA M.; CAROLI S. Analytical methods for the quantification of volatile aromatic compounds. TrAC Trends in Analytical Chemistry, 30(11), 1756-1770. 2011.

Steffens, C.; Franceschi, E.; Corazza, F.C.; Herrmann, P.S.P.; Oliveira, J.V. Gas sensor development using supercritical fluid technology to detect the ripeness of bananas. J. Food Eng. 2010, 101, 365-369.

BANERJEE R.; TUDU B.; SHAW L.; JANA A.; BHATTACHARYYA N.; BANDYOPADHYAY R. Instrumental testing of tea by combining the responses of electronic nose and tongue. Journal of Food Engineering, 110(3), 356-363. 2012.

GUISEPPI-ELIE A.G.; WALLACE G.G.; MATSUE T.; in: T.A. Skotheim, R.L. Elsenbaumer, J.R. Reynolds (Eds.). Handbook of Conducting Polymers, Marcel Dekker, New York, 1998, p. 963.

LI Z. F.; BLUM F. D.; BERTINO M. F.; KIM C. S. Understanding the response of nanostructured polyaniline gas sensors. Sensors and Actuators B: Chemical, 183, 419-427. 2013.

VENANCIO E. C.; MATTOSO L. H. C.; HERRMANN P. S. D. P.; MACDIARMID A. G. Line patterning of graphite and the fabrication of cheap, inexpensive, "throw-away" sensors. Sensors and Actuators B: Chemical, 130(2), 723-729. 2008.

WYLLIE S.G. Flavour quality of fruit and vegetables: are we on the brink of major advances In: Brückner, B., Wyllie, S.G. (Eds.), Fruit and Vegetable Flavour: recent Advances and Future Prospects. CRC Press, Boca Raton. 2008.

HAWARI H. F.; SAMSUDIN, N. M.; AHMAD M. N.; SHAKAFF A. Y. M.; GHANI, S. A.; WAHAB Y.; AKITSU T. Array of MIP-Based Sensor for Fruit Maturity Assessment. Procedia Chemistry, 6, 100-109. 2012.

VOILLEY A.; SOUCHON I. Flavour retention and release from food matrix: an overview. In Voilley A., Etievant P. (Eds.) Flavour in foods. Cambridge: Woodhead Publishing Ltd; 117 132. 2006.

STEFFENS C., CORAZZA M. L., FRANCESCHI E., CASTILHOS F., HERRMANN P. S. P., Oliveira, J. V. Development of gas sensors coatings by polyaniline using pressurized fluid. Sensors and Actuators B: Chemical, 171-172, 627-633. 2012.

WEBER I. T.; LEITE E. R.; LONGO E.; VARELA J. A.; LIEC U.D.; LUIZ R. W. Development of gas sensors based on nanostructured $\mathrm{SnO} 2$ : the influence of microstructrure on sensors performance. 46(299), 156-159. 2000. 